\title{
Time-Delay Estimation by Enhanced Orthogonal Matching Pursuit Method for Thin Asphalt Pavement With Similar Permittivity
}

\author{
Meng Sun ${ }^{(}$, Jingjing Pan $^{(}$, Member, IEEE, Yide Wang ${ }^{(\mathbb{1}}$, Senior Member, IEEE, \\ Xiaofei Zhang ${ }^{\circledR}$, Xiaoting Xiao, Cyrille Fauchard, and Cédric Le Bastard
}

\begin{abstract}
Time-delay estimation (TDE) for thin top layers of asphalt pavement is a challenging task due to the limited resolution of ground penetrating radar (GPR) as well as small permittivity difference between top layers. Echoes backscattered from the interfaces of top layers with similar permittivity have usually much smaller amplitudes compared with other echoes, which can be called weak signals. The weak backscattered echoes are usually too sensitive to the noise and other strong echoes that current signal processing approaches (subspace-based methods and compressed sensing based methods) might have false estimation results even failures without proper processing of them. Therefore, in this paper, an enhanced orthogonal matching pursuit (OMP) method is proposed to deal with weak signals resulting from similar permittivity of adjacent asphalt layers. Based on the orthogonality between signal and noise subspaces, we firstly apply the truncated singular value decomposition (SVD) on the received signals, in order to reduce the noise impact. Secondly, we build an orthogonal matrix to the mode matrix of the pre-estimated strong backscattered echoes, and map it to the overcomplete dictionary matrix, such that the influence of the residual of the strong backscattered echoes can be reduced. Finally, the time-delays of backscattered echoes and layer thicknesses are estimated. Compared with conventional approaches, the proposed method is more suitable for TDE in thin asphalt pavement detection. The accuracy of the proposed method is validated by both numerical and experimental data.
\end{abstract}

Index Terms-Pavement survey, time-delay estimation (TDE), thickness measurement, permittivity, orthogonal matching pursuit (OMP).

Manuscript received October 6, 2020; revised April 11, 2021; accepted May 18, 2021. This work was supported in part by the Key Laboratory of Dynamic Cognitive System of Electromagnetic Spectrum Space, Ministry of Industry and Information Technology, Nanjing University of Aeronautics and Astronautics, under Grant KF20202101 and Grant KF20202109, in part by the National Natural Science Foundation of China under Grant 61701085, and in part by the Fundamental Research Funds for the Central Universities under Grant NS2021028. The Associate Editor for this article was C. F. Mecklenbräuker. (Corresponding author: Jingjing Pan.)

Meng Sun, Jingjing Pan, and Xiaofei Zhang are with the Department of Electronic Engineering, Nanjing University of Aeronautics and Astronautics, Nanjing 211106, China, and also with the Key Laboratory of Dynamic Cognitive System of Electromagnetic Spectrum Space (Nanjing University of Aeronautics and Astronautics), Ministry of Industry and Information Technology, Nanjing 211106, China (e-mail: jingjingpan@nuaa.edu.cn).

Yide Wang is with the IETR, University of Nantes, 44306 Nantes, France. Xiaoting Xiao is with the School of Electrical Engineering and Information, Southwest Petroleum University, Chengdu 610599, China.

Cyrille Fauchard is with the ENDSUM, CEREMA, 49136 Angers, France.

Cédric Le Bastard resides in 49136 Angers, France.

Digital Object Identifier 10.1109/TITS.2021.3088312

\section{INTRODUCTION}

A SPHALT pavement survey is an important task for the condition rating, quality management, and maintenance of pavements in civil engineering [1]-[3]. Direct ways for the structural health monitoring and state evaluation, like core drilling, are usually destructive, time consuming and cost intensive, and the evaluation is only conducted over limited sample points. Non-destructive testing (NDT) methods are promising by allowing analysis of the properties of a material without destroying the serviceability of the original part. As a typical geophysical NDT method, ground penetrating radar (GPR) has become an established and routine approach of road structure survey with a more extensive measurement coverage in a rapider manner, compared with traditional coring ones [4]-[10]. The vertical structure of pavements can be deduced from radar profiles by means of echo detection and amplitude estimation. Echo detection provides the time-delay associated with each interface, while amplitude estimation is used to retrieve the wave speed within each layer.

On the fundamental level, GPR instruments transmit radio waves into pavement and detect the backscattered echoes from changes within the pavement structure. The resolution scale of GPR systems should be in good match for the scale of assessment for the sake of accuracy. In case of thin layers, where backscattered echoes are overlapped and indistinguishable in terms of GPR wavelength, a proper design of GPR instruments or high resolution techniques are in high demand. In addition, a further issue concerns the interpretation of GPR data. An effective pavement survey and diagnose requires a massive amount of data, while for intelligent transportation systems, the evaluation is preferred to operate automatically or in real time. GPR data interpretation is highly subjective to user experience and knowledge [11]. Therefore, intelligent inspections or advanced signal processing techniques are needed in pavement survey by providing both correct and efficient information for either operators or end users (clients or awarding authorities) to optimize the road structure's maintenance.

Over the years, a variety of intelligent signal processing methods have been proposed to facilitate the interpretation of GPR data, including subspace-based methods, compressive sensing based methods, and intelligent learning methods and so on [12]-[15]. In the work of [16]-[18], subspace based methods, or high resolution methods, show their great potential in dealing with overlapped backscattered echoes, especially in the survey of thin layers. It should be noted 
that subspace based methods require additional rank-restore preprocessing procedures for the proper split of signal and noise subspace, because of the coherency of backscattered echoes. In [17], [19]-[21], the authors propose to use spatial smoothing preprocessing techniques and their improved variants for decorrelation. In addition, compressive sensing based methods, or sparse representation based methods, exploit the sparsity of signals in the interpretation of GPR data. In [22], [23], second order cone programming and orthogonal matching pursuit (OMP) are applied in the thickness estimation of pavement, and achieve good results in time-delay estimation (TDE). Unlike subspace based methods, compressive sensing based methods allow direct processing of coherent signals without applying spatial smoothing.

There is also an increasing demand in using intelligent machine learning methods in GPR application, owning to the remarkable performance in data analysis. A classical learning process is followed by preprocessing (noise elimination, data representation), feature extraction, detection/recognition and so on [12], [24]-[26]. The learning is usually conducted by establishing a high nonlinear mapping between the features and the recognition results. In [12], a support vector machines (SVM) based method is proposed for TDE in the pavement survey, which offers excellent performance for GPR data interpretation in the scenario of small number of snapshots or even a single snapshot. Artificial neural networks have also been extensively applied to improve the interpretation of GPR signals or images, especially with the explosion of deep learning and big data. The authors in [25] propose a deep learning based pavement defect detection method; the proposed method detects the pavement distresses accurately, which is be affected by GPR transmitting frequencies. Nevertheless, intelligent learning based methods are highly dependent on the training data for the desired model [27].

Apart from the thin layer problem, another factor limiting the accuracy of the thickness estimation is the permittivity of the pavement materials [28]. In asphalt pavements, the materials in the sub-layers are similar that there is no significant permittivity difference between adjacent asphalt layers [29], [30]. Therefore, the backscattered echoes at the interfaces of sub-layers have much smaller amplitude than that of the surface layers, which can be called weak signal. The detection of weak signals is easily affected by noise or strong backscattered echoes, which is therefore suffering from performance degradation even failures in scenarios of overlapping signals and low signal to noise ratio (SNR). The detection errors of weak signal will lead to a significant decrease in the accuracy of TDE as well as thickness estimation. However, few has been done in the literature for TDE in the presence of small permittivity difference between two adjacent layers. The authors in [31] propose a weak signal detection method by using Hilbert transform. Nevertheless, this method can only work in scenarios of non-overlapped echoes. TDE for asphalt layers remains to be a challenging task considering the time resolution and weak reflection.

Motivated by the aforementioned thin layer and weak signal detection problems, we propose an enhanced OMP method for TDE in an efficient and automatic manner to evaluate the thin asphalt pavement with similar permittivity for intelligent transportation systems. The proposed method consists of three steps. Firstly, the truncated singular value decomposition (SVD) is applied to reduce the noise impact to the backscattered echoes, especially to weak signals. Secondly, an orthogonal matrix to the mode matrix of the pre-estimated strong backscattered echoes is constructed to eliminate the influence of strong echoes to weak ones. The pre-estimation is performed by using OMP procedures. Finally, the enhanced OMP is used to estimate the times of arrival of weak signals. The main contributions of this paper are: 1) reducing the noise impact by applying the truncated SVD on the received signal; 2) eliminating the influence of the residual of the strong backscattered echoes by constructing a matrix that is orthogonal to the mode matrix of the pre-estimated strong backscattered echoes. The proposed method estimates the time-delay and thickness of thin asphalt pavement from different points of roadway in real time, which is efficient and accurate for GPR data interpretation in pavement survey and meets the needs of intelligent transportation systems. The estimated time-delay and thickness provide important information for human or automated decision making for intelligent transportation systems. The performance of the proposed method is tested on both numerical and experimental data. The testing results show the efficiency and accuracy of the proposed method in thin asphalt pavement evaluation.

The rest of the paper is organized as follows: Section II presents the methodology of this paper, where the signal model, the proposed enhanced OMP method for TDE in presence of weak signals are introduced. The numerical and experimental results of the proposed enhanced OMP method are provided in Sections III and IV, respectively. Conclusions are drawn in section $\mathrm{V}$.

\section{Methodology}

\section{A. Signal Model}

In this work, we consider the top layers of asphalt pavement, which can be regarded as low-loss media [32]. As described in [33], the dispersivity of the low-loss media is negligible. Therefore, the received radar signal can be approximately expressed as the time-shifted and attenuated copies of the transmitted signal [19]-[21], [34]:

$$
\begin{aligned}
r(f)= & \sum_{x=1}^{X} e(f) s_{x} \exp \left(-j 2 \pi f t_{x}\right) \\
& +\sum_{y=1}^{Y} e(f) s_{y} \exp \left(-j 2 \pi f t_{y}\right)+n(f) \\
= & \sum_{k=1}^{K} e(f) s_{k} \exp \left(-j 2 \pi f t_{k}\right)+n(f),
\end{aligned}
$$

where

- $X$ is the number of strong backscattered echoes; $Y$ represents the number of echoes backscattered from interfaces of asphalt layers with similar permittivity (weak signals); $K=X+Y$ is the total number of backscattered echoes, which is assumed to be known;

- $e(f)$ is the radar pulse in frequency domain;

- $s_{x}$ and $s_{y}$ represent the amplitudes of the $x$ th strong backscattered echo and $y$ th weak signal, respectively. For low-loss media, the amplitude of the backscattered echo is dependent on the reflection coefficient of the media, which can be assumed to be real; 


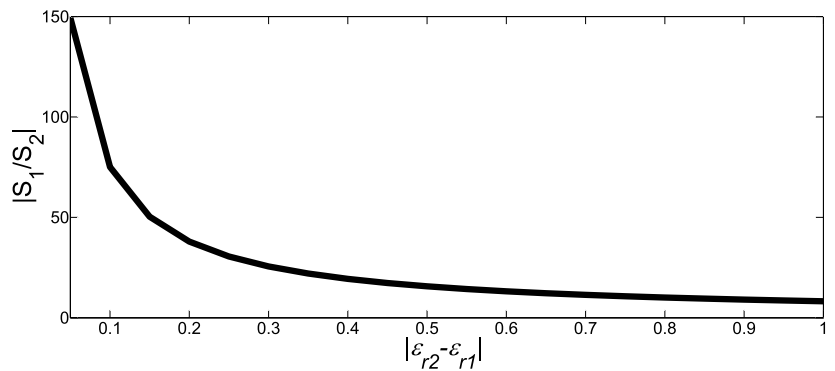

Fig. 1. The ratio between the amplitudes of $S_{1}$ and $S_{2}$ versus $\left|\varepsilon_{r 2}-\varepsilon_{r 1}\right|$ under noiseless circumstance.

- $n(f)$ is an additive white Gaussian noise with zero mean and variance $\sigma^{2}$.

Applying the frequency-domain sampling $\left(f_{i}=f_{1}+(i-\right.$ 1) $\Delta f$ and $i=1,2 \ldots N)$ to (1) with the number of used frequencies $N$, the lowest frequency $f_{1}$ and the frequency step $\Delta f$, the radar data model can be reformulated in the following vector form:

$$
\mathbf{r}_{v}=\mathbf{\Lambda} \mathbf{A}_{X} \mathbf{s}_{X}+\mathbf{\Lambda} \mathbf{A}_{Y} \mathbf{s}_{Y}+\mathbf{n}=\mathbf{\Lambda} \mathbf{A} \mathbf{s}+\mathbf{n},
$$

where

- $\mathbf{r}_{v}=\left[r\left(f_{1}\right) r\left(f_{2}\right) \cdots r\left(f_{N}\right)\right]^{T}$ is the $(N \times 1)$ received signal vector representing the measured GPR signal in frequency domain or the measurements by a step-frequency GPR; superscript $T$ denotes the transpose operation;

- $\boldsymbol{\Lambda}=\operatorname{diag}\left\{e\left(f_{1}\right), e\left(f_{2}\right), \ldots, e\left(f_{N}\right)\right\}$ is a $(N \times N)$ diagonal matrix containing the frequency information of the radar pulse;

- $\mathbf{A}=\left[\mathbf{A}_{X}, \mathbf{A}_{Y}\right]=\left[\mathbf{a}\left(t_{1}\right) \mathbf{a}\left(t_{2}\right) \cdots \mathbf{a}\left(t_{K}\right)\right]$ is the mode matrix;

- $\mathbf{a}\left(t_{k}\right)=\left[e^{-2 j \pi f_{1} t_{k}} e^{-2 j \pi f_{2} t_{k}} \ldots e^{-2 j \pi f_{N} t_{k}}\right]^{T}$ is the mode vector of the $k$ th backscattered echo;

- $\mathbf{s}=\left[\mathbf{s}_{X}, \mathbf{s}_{Y}\right]^{T}=\left[\begin{array}{llll}s_{1} & s_{2} & \cdots & s_{K}\end{array}\right]^{T}$ is the $(K \times 1)$ vector of the amplitudes of backscattered echoes;

- $\mathbf{n}=\left[n\left(f_{1}\right) n\left(f_{2}\right) \cdots n\left(f_{N}\right)\right]^{T}$ is the $(N \times 1)$ noise vector.

According to the rules of Fresnel reflection, the amplitude of the backscattered echo is dependent on the permittivity of adjacent layers that are separated by the corresponding interface. The greater the difference between adjacent layers is, the larger the amplitude of the backscattered echo at the interface is. In the scenario of asphalt pavement, the permittivities of the surface layer and its adjacent layers are similar; thus, the echo backscattered from the interface of the first two layers has much smaller amplitude compared to the other backscattered echoes.

Under noiseless circumstance, a simulation example about the ratio between the amplitudes of two backscattered echoes versus the permittivity difference of the first two layer of an asphalt pavement is presented. $S_{1}$ and $S_{2}$ represent echoes backscattered from surface layer (permittivity $\varepsilon_{r 1}$ ) and its adjacent layer (permittivity $\varepsilon_{r 2}$ ), respectively. Permittivity $\varepsilon_{r 1}$ is fixed at $4.5, \varepsilon_{r 2}=\varepsilon_{r 1}+\Delta \varepsilon, \Delta \varepsilon \in[0.05,1]$. As shown in Fig. 1, the ratio between the amplitudes of $S_{1}$ and $S_{2}$ is continuously decreasing with the increasing of the permittivity difference of the adjacent layers. When the $\left|\varepsilon_{r 2}-\varepsilon_{r 1}\right| \leq 0.4$, the ratio is greater than 20. In this situation, $S_{2}$ can be considered as a weak signal. It is very difficult to detect the echo backscattered from the interface of layers with similar permittivity (weak signal), especially for thin asphalt layers, due to the influence of the noise and strong backscattered echoes.

To solve the problem caused by the tiny permittivity difference between adjacent asphalt layers, in the following, an enhanced OMP method is proposed for TDE in presence of weak signals.

\section{B. SVD for Denoising Procedure}

To begin with, the truncated SVD is applied on the received signal model (2) with number of snapshots $N_{x}$ :

$$
\mathbf{R}=\left[\mathbf{r}_{1}, \mathbf{r}_{2}, \ldots, \mathbf{r}_{N_{x}}\right]=\mathbf{U} \boldsymbol{\Phi} \mathbf{V}^{H},
$$

where $\mathbf{r}_{i}$ is the $i$ th snapshot of the received signal, $\boldsymbol{\Phi}$ is a $(N \times N)$ diagonal matrix whose diagonal elements are non-zero singular values of $\mathbf{R}$; the $(N \times N)$ dimensional matrix $\mathbf{U}$ and $\left(N_{x} \times N\right)$ dimensional matrix $\mathbf{V}$ are the left and right singular vector matrices of $\mathbf{R}$, respectively. Matrix $\mathbf{R}$ can be divided into the following two parts:

$$
\begin{aligned}
\mathbf{R} & =\mathbf{R}_{s}+\mathbf{R}_{n} \\
& =\left[\mathbf{U}_{s}, \mathbf{U}_{n}\right]\left[\begin{array}{cc}
\boldsymbol{\Phi}_{s} & 0 \\
0 & \boldsymbol{\Phi}_{n}
\end{array}\right]\left[\begin{array}{c}
\mathbf{V}_{s}^{H} \\
\mathbf{V}_{n}^{H}
\end{array}\right] \\
& =\mathbf{U}_{s} \boldsymbol{\Phi}_{s} \mathbf{V}_{s}^{H}+\mathbf{U}_{n} \boldsymbol{\Phi}_{n} \mathbf{V}_{n}^{H},
\end{aligned}
$$

where $\mathbf{R}_{s}$ and $\mathbf{R}_{n}$ are associated with the signal and noise, respectively. $\boldsymbol{\Phi}_{s}$ and $\boldsymbol{\Phi}_{n}$ are the diagonal matrix with the $K$ largest and $N-K$ smaller non-zero singular values of $\mathbf{R}$, respectively. $\left(\mathbf{U}_{s}, \mathbf{V}_{s}\right)$ and $\left(\mathbf{U}_{n}, \mathbf{V}_{n}\right)$ are the singular vector matrices corresponding to the $K$ dominant singular values and $N-K$ smaller singular values, respectively. In GPR measurements, the backscattered echoes are coherent, therefore, $\boldsymbol{\Phi}_{s}$ is a scaler; $\mathbf{U}_{s}$ and $\mathbf{V}_{s}$ are $(N \times 1)$ and $\left(N_{x} \times 1\right)$ vectors, respectively. Therefore, $\mathbf{R}_{s}$ can be reformulated as

$$
\mathbf{R}_{s}=\phi_{s} \mathbf{u}_{s} \mathbf{v}_{s}^{H} .
$$

By using the orthogonality between the signal and noise subspaces, the received signal is enhanced by $\mathbf{R}_{s}$ such that the impact of the additive noise can be reduced. The enhanced signal model can then be expressed as

$$
\begin{aligned}
\mathbf{R}_{e n} & =\mathbf{R}_{s} \mathbf{R}_{s}^{H} \mathbf{R}=\mathbf{R}_{s} \mathbf{R}_{s}^{H}\left(\mathbf{R}_{s}+\mathbf{R}_{n}\right) \\
& =\phi \mathbf{u}_{s} \mathbf{v}_{s}^{H} .
\end{aligned}
$$

In the following, the radar pulse (matrix) $\boldsymbol{\Lambda}$ should be removed before the application of the proposed method. Therefore, the whitening procedure is used. The received signal model can then be expressed as $\mathbf{r}_{e n}=\boldsymbol{\Lambda}^{-1} \mathbf{r}$, where the elements of $\mathbf{r}$ correspond to the mean values of each row of $\mathbf{R}_{e n}$.

\section{Enhanced Orthogonal Matching Pursuit Method}

With the enhanced signal model, the overcomplete dictionary matrix $\mathbf{A}_{t}$ is built. The entire time domain of GPR working time window is sampled at $T=\left[\begin{array}{lll}\tau_{1} & \tau_{2} \ldots \tau_{N_{t}}\end{array}\right]$, with $N_{t} \gg K$. Accordingly, the $\left(N \times N_{t}\right)$-dimensional dictionary matrix can be expressed as $\mathbf{A}_{t}=\left[\mathbf{a}\left(\tau_{1}\right), \mathbf{a}\left(\tau_{2}\right), \ldots, \mathbf{a}\left(\tau_{N_{t}}\right)\right]$. Similar to the conventional OMP method, the enhanced OMP estimates the times of arrival of the backscattered echoes through two steps. The first step is to select the best fitting atom from the dictionary matrix $\mathbf{A}_{t}$; the second step is to calculate the amplitude of echoes and signal residual for the 
following iteration. It should be emphasized that the number of iterations is equal to the number of backscattered echoes.

The best fitting atom is the one that most strongly correlates with the signal residual which provides the estimated time of arrival at each iteration. For the conventional OMP, in the $k$ th iteration, the correlation between the signal residual $\mathbf{r}_{e n}^{k-1}$ (the initial signal residual is set to be $\mathbf{r}_{e n}$ ) and atoms of dictionary matrix $\mathbf{A}_{t}$ is defined as

$$
\mu_{k}(i)=\left|\mathbf{a}^{H}\left(\tau_{i}\right) \mathbf{r}_{e n}^{k-1}\right| .
$$

In the signal residual of the $k$ th iteration, the first $k-1$ strong backscattered echoes are deleted theoretically, but in practice, the residual of these echoes will still have influence on echo detection in the following iterations, especially for the weak signals. Therefore, in the $k$ th iteration, to minimize or suppress the influence of the residual of the $k-1$ strong backscattered echoes to the weak signals, the following approach is proposed. In the first $k-1$ iterations, the mode matrix of the $k-1$ strong backscattered echoes $\mathbf{A}_{k-1}=\left[\mathbf{a}\left(\hat{t}_{1}\right), \ldots, \mathbf{a}\left(\hat{t}_{k-1}\right)\right]$ is obtained. Then, its orthogonal projection matrix $\overline{\mathbf{A}}_{k-1}$ can be constructed as

$$
\overline{\mathbf{A}}_{k-1}=\mathbf{I}-\mathbf{A}_{k-1}\left(\mathbf{A}_{k-1}^{H} \mathbf{A}_{k-1}\right)^{-1} \mathbf{A}_{k-1}^{H},
$$

with I the $N \times N$ identity matrix. Multiplying $\overline{\mathbf{A}}_{k-1}$ by the signal residual $\mathbf{r}_{e n}^{k-1}$ of the $k$ th iteration, the influence of strong backscattered echoes can then be eliminated.

Therefore, in the $k$ th iteration, the proposed enhanced OMP calculates the correlation between the signal residual $\mathbf{r}_{e n}^{k-1}$ and dictionary matrix $\mathbf{A}_{t}$ as follows:

$$
\mu_{k}(i)=\left|\mathbf{a}^{H}\left(\tau_{i}\right) \overline{\mathbf{A}}_{k-1} \mathbf{r}_{e n}^{k-1}\right| .
$$

The best-fitting atom corresponds to the atom with the highest correlation $\mu_{k}(i)$. Because echoes are backscattered from low-loss media, their amplitudes can be assumed to be real. Likewise, when $\tau_{i}=t_{k}, \mathbf{a}^{H}\left(\tau_{i}\right) \overline{\mathbf{A}}_{k-1} \mathbf{r}_{e n}^{k-1}$ represents the estimated amplitude of the $k$ th backscattered echo after eliminating the $k-1$ backscattered echoes, should be theoretically real. Accordingly, only real part of $\mu_{k}(i)$ is required in the best fitting atom selection, and (9) can be reformulated as

$$
\mu_{k}(i)=\left|\operatorname{real}\left\{\mathbf{a}^{H}\left(\tau_{i}\right) \overline{\mathbf{A}}_{k-1} \mathbf{r}_{e n}^{k-1}\right\}\right|,
$$

the time of arrival $\hat{t}_{k}$ can then be estimated by finding the best fitting atom.

In the second step, with the selected best fitting atom in the $k$ th iteration, the amplitude of the $k$ th backscattered echo is estimated by the following LS solution:

$$
\hat{s}_{k}=\mathbf{a}^{+}\left(\hat{t}_{k}\right) \mathbf{r}_{e n}^{k-1},
$$

where operator + denotes the Moore-Penrose transpose. Afterwards, the signal residual for the $(k+1)$ th iteration is calculated as

$$
\mathbf{r}_{e n}^{k}=\mathbf{r}_{e n}^{k-1}-\mathbf{a}\left(\hat{t}_{k}\right) \hat{s}_{k}
$$

The detail of the proposed enhanced OMP method is summarized in Table I. By using the proposed method, the weak signal can be detected; consequently, the times of arrival of both strong and weak backscattered echoes are estimated.
TABLE I

Procedure of THE ENHANCED OMP Method

\section{INPUT:}

$\left(N \times N_{t}\right)$-dimensional overcomplete dictionary matrix $\mathbf{A}_{t}$

$(N \times 1)$-dimensional received signal $\mathbf{r}_{e n}$

The number of backscattered echoes $K$

$X$ strong and $Y$ weak echoes, $K=X+Y$

OUTPUT:

Estimated times of arrival $\hat{t}_{k}, k=1,2, \ldots, K$

\section{INITIALIZATION:}

Initial signal residual $\mathbf{r}_{e n}^{0}=\mathbf{r}_{e n}$

Initial iteration step $k=1$

Initial index set $\Psi^{0}=\emptyset$

Initial mode matrix of selected atoms $\mathbf{A}_{0}=\emptyset$

Initial projection matrix $\overline{\mathbf{A}}_{0}=\mathbf{I}$

while not converged $(k<K)$ do

\section{MATCH:}

$\mu_{k}(i)=\left|\operatorname{real}\left\{\mathbf{a}^{H}\left(\tau_{i}\right) \overline{\mathbf{A}}_{k-1} \mathbf{r}_{e n}^{k-1}\right\}\right|, i=1,2, \ldots, N_{t}$

$\hat{t}_{k}$ can be estimated by solving the optimization problem:

$\hat{t}_{k}=\underset{i}{\arg \max }\left\{\mu_{k}(i)\right\}$

\section{UPDATE:}

Amplitude of the $k$ th echo $\hat{s}_{k}=\mathbf{a}^{+}\left(\hat{t}_{k}\right) \mathbf{r}_{e n}^{k-1}$

Signal residual $\mathbf{r}_{e n}^{k}=\mathbf{r}_{e n}^{k-1}-\mathbf{a}\left(\hat{t}_{k}\right) \hat{s}_{k}$

Iteration step $k=k+1$

Index set $\boldsymbol{\Psi}^{k}=\boldsymbol{\Psi}^{k-1} \cup \hat{t}_{k}$

Mode matrix of selected atoms $\mathbf{A}_{k}=\left[\mathbf{A}_{k-1}, \mathbf{a}\left(\hat{t}_{k}\right)\right]$

Projection matrix $\overline{\mathbf{A}}_{k}=\mathbf{I}-\mathbf{A}_{k}\left(\mathbf{A}_{k}^{H} \mathbf{A}_{k}\right)^{-1} \mathbf{A}_{k}^{H}$

\section{end while}

\section{NUMERICAL RESULTS}

In this section, the performance of the proposed enhanced OMP method is tested by 3 simulations with the numerical data obtained from (1). It can be seen from Fig. 2 that the studied pavement is made up of three homogeneous layers. The first layer is an ultra-thin asphalt surfacing (UTAS) with relative permittivity $\varepsilon_{r 1}=4.5$; the second layer is also made of asphalt, whose relative permittivity is very close to that of the first layer. The third layer is a base layer. $H_{1}, H_{2}$ and $\mathrm{H}_{3}$ correspond to the thicknesses of Layer 1, Layer 2, and Layer 3, respectively. Therefore, the echo backscattered from the second interface has much smaller amplitude compared to the first and third echoes. In the following, the used frequency band is $B \in[0.5,2.5] \mathrm{GHz}$, with 21 frequency samples $(0.1 \mathrm{GHz}$ frequency step). The number of independent snapshots is 500. SNR is defined as the ratio between the powers of the echo backscattered from Layer 1 and the noise variance.

\section{ACCEPTED MANUSCRIPT}




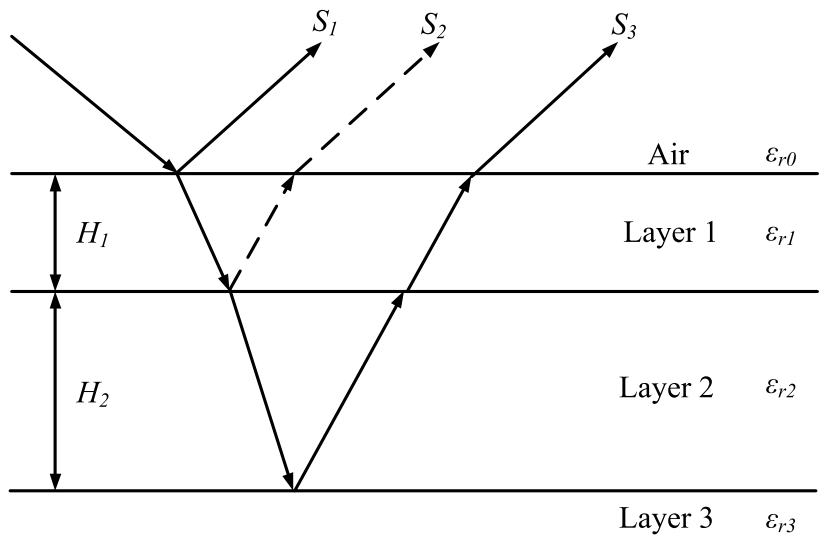

Fig. 2. Pavement configuration.

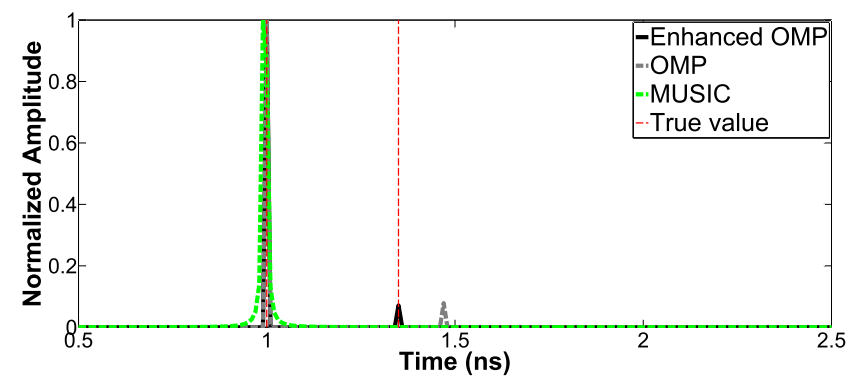

Fig. 3. Case 1: TDE with $\mathrm{SNR}=10 \mathrm{~dB}$, the red dashed lines represent the true times of arrival, two overlapped echoes.

\section{A. Performance Under Different Pavement Parameters}

In the first simulation, the performance of the proposed enhanced OMP method is tested and compared with that of the conventional OPM and MUSIC (modified spatial smoothing is used for the decorrelation procedure with 10 subbands) in a single run of the algorithms. SNR is fixed at $10 \mathrm{~dB}$ for the different pavements. Pavements with different layers, permittivities and thicknesses are considered for both overlapped and non-overlapped echoes:

- Case 1. Two backscattered echoes; $H_{1}=25 \mathrm{~mm}$ and $H_{2}=H_{3}=\infty ; \varepsilon_{r 2}=\varepsilon_{r 3}=4.7$; the corresponding times of arrival $\left(t_{1}, t_{2}\right)$ are $1.00 \mathrm{~ns}$ and $1.35 \mathrm{~ns}$, respectively. The two echoes are overlapped.

- Case 2. Three backscattered echoes; $H_{1}=40 \mathrm{~mm}$, $H_{2}=45 \mathrm{~mm}$ and $H_{3}=\infty ; \varepsilon_{r 2}=4.7, \varepsilon_{r 3}=7$; the corresponding times of arrival $\left(t_{1}, t_{2}, t_{3}\right)$ are $1.00 \mathrm{~ns}$, $1.57 \mathrm{~ns}$ and $2.22 \mathrm{~ns}$, respectively. The three echoes are non-overlapped.

- Case 3. Three backscattered echoes; $H_{1}=25 \mathrm{~mm}$, $H_{2}=20 \mathrm{~mm}$ and $H_{3}=\infty ; \varepsilon_{r 2}=4.7, \varepsilon_{r 3}=7$; the corresponding times of arrival $\left(t_{1}, t_{2}, t_{3}\right)$ are $1.00 \mathrm{~ns}$, $1.35 \mathrm{~ns}$ and $1.64 \mathrm{~ns}$, respectively. The three echoes are overlapped.

As shown in Figs. 3-5, the times of arrival of the backscattered echoes from 3 different pavements can be well estimated by the enhanced OMP method for both overlapped and non-overlapped echoes. The estimated times of arrival and amplitudes of the backscattered echoes corresponding to the abscissas and ordinates of the peaks, respectively, are clearly detected. The echo backscattered from the interface of two layers with similar permittivity is a weak signal, whose amplitude is small. Therefore, the peak corresponding to the

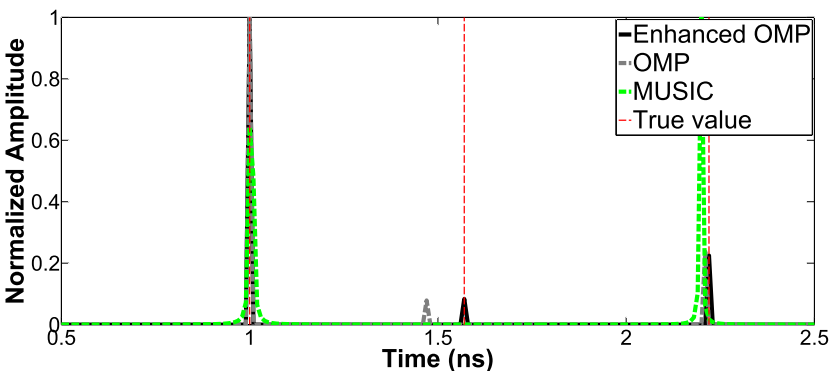

Fig. 4. Case 2: TDE with $\mathrm{SNR}=10 \mathrm{~dB}$, the red dashed lines represent the true times of arrival, three non-overlapped echoes.

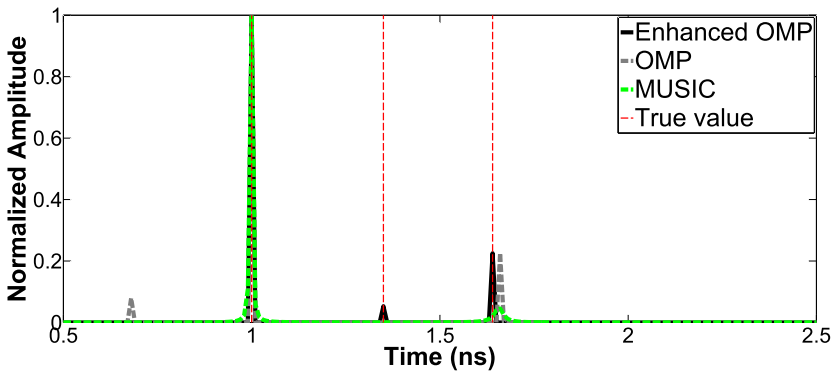

Fig. 5. Case 3: TDE with $\mathrm{SNR}=10 \mathrm{~dB}$, the red dashed lines represent the true times of arrival, three overlapped echoes.

TABLE II

Simulation Parameters Applied in Case 4 (4 Echoes BACKSCATTERED FROM 4 INTERFACES)

\begin{tabular}{|c|c|c|c|c|}
\hline Layer/Interface & 1 & 2 & 3 & 4 \\
\hline Parameter & 4.5 & 4.7 & 6.8 & 7 \\
\hline Permittivity of layer & 2.5 & 3.0 & 4.0 & $\infty$ \\
\hline Thickness of layer (cm) & 1.00 & 1.35 & 1.78 & 2.48 \\
\hline Time of arrival (ns)
\end{tabular}

weak signal is with low height, see Figs. 3-5. However, due to the limited resolution, MUSIC algorithm fails in the detection of the weak signal (backscattered from the interface of two layers with similar permittivity), while the conventional OMP estimates the time of arrival of the weak signal with bias.

The proposed enhanced OMP can also be used to detect more than one weak signal, as mentioned in the following case (Case 4). In Case 4, 4 echoes backscattered from a pavement made up of 4 interfaces (4 layers) are considered. The pavement parameters are shown in Table II. Echoes backscattered from the second and fourth interfaces can be regarded as weak signals. Fig. 6 plots the estimated times of arrival of the 4 backscattered echoes. It can be seen that both the conventional OMP and MUSIC fail to detect the weak signals (the second and fourth echoes). Nevertheless, the proposed method still works in this scenario. The estimated times of arrival of the weak signals by the proposed method are very close to the true values, which means that the enhanced OMP can resolve more than one weak signal.

\section{B. Performance Versus Permittivity Difference Between Adjacent Layers}

In the second simulation, the ability of the enhance OMP for weak signal detection is studied. The performance of the proposed method versus the permittivity difference between adjacent layers $\left|\varepsilon_{r 2}-\varepsilon_{r 1}\right|$ (Layers 1 and 2 ) for both overlapped and non-overlapped echoes is evaluated with 200 independent 


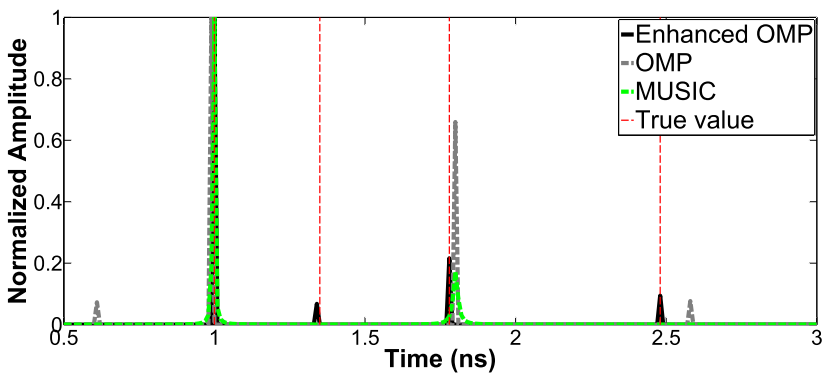

Fig. 6. Case 4: TDE with SNR $=10 \mathrm{~dB}$, the red dashed lines represent the true times of arrival, four echoes.

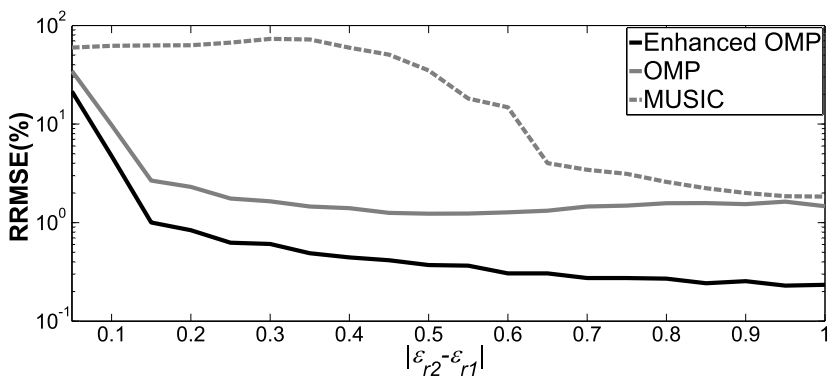

Fig. 7. RRMSE on the estimated time of arrival $\hat{t}_{2}$ of weak signal $S_{2}$ versus $\left|\varepsilon_{r 2}-\varepsilon_{r 1}\right|$ with 200 Monte-Carlo processes. $H_{1}=40 \mathrm{~mm}$ and $H_{2}=H_{3}=\infty$, non-overlapped echoes.

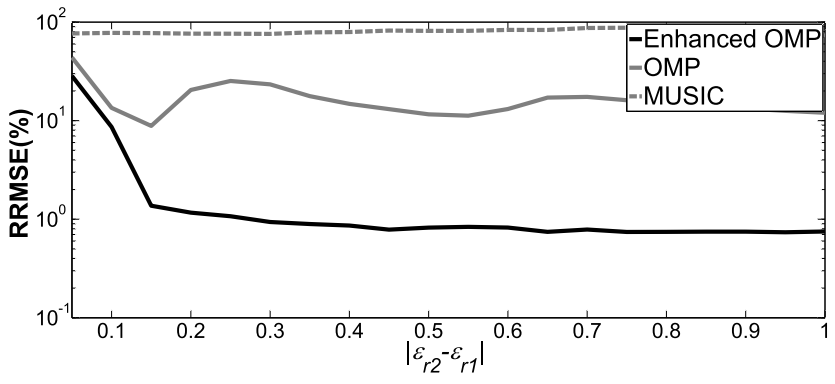

Fig. 8. RRMSE on the estimated time of arrival $\hat{t}_{2}$ of weak signal $S_{2}$ versus $\left|\varepsilon_{r 2}-\varepsilon_{r 1}\right|$ with 200 Monte-Carlo processes. $H_{1}=25 \mathrm{~mm}$ and $H_{2}=H_{3}=\infty$, overlapped echoes.

runs of the method. The permittivity of Layer $1 \varepsilon_{r 1}$ is fixed at 4.5 , while the permittivity of Layer 2 is set as $\varepsilon_{r 2}=\varepsilon_{r 1}+$ $\Delta \varepsilon, \Delta \varepsilon \in[0.05,1]$. SNR is fixed at $10 \mathrm{~dB}$. The relative root-mean-squared error (RRMSE) on the estimated time of arrival $\hat{t}_{2}$ of weak signal $S_{2}$ is calculated.

Figs. 7-8 show the RRMSEs on the estimated $\hat{t}_{2}$ of weak signal $S_{2}$ by using the enhanced OMP, conventional OMP and MUSIC. As can be seen from Fig. 7, for non-overlapped echoes, RRMSEs on $\hat{t}_{2}$ is decreasing when $\left|\varepsilon_{r 2}-\varepsilon_{r 1}\right|$ increases for the compared three methods. The proposed method has better accuracy than the conventional OMP and MUSIC. For overlapped echoes, as illustrated by Fig. 8, both the conventional OMP and MUSIC fail to detect the weak signal $S_{2}$. Nevertheless, the proposed method remains robust for TDE.

\section{Performance Versus SNR}

In order to test the ability of the proposed enhanced OMP method with respect to noise, in the third simulation, the statistics performance of the proposed method versus SNR is evaluated with 200 Monte-Carlo processes. The RRMSE on the estimated parameter $t_{2}$ is calculated. Cases 2 and 3

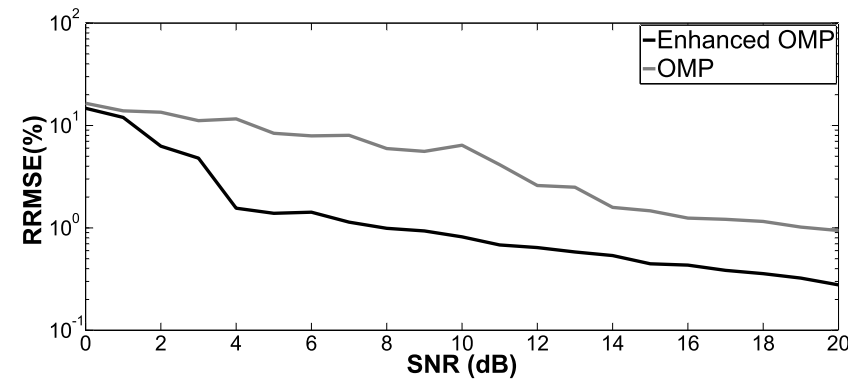

Fig. 9. Case 2, RRMSE on the estimated time of arrival $\hat{t}_{2}$ of weak signal $S_{2}$ versus SNR with 200 Monte-Carlo processes, non-overlapped echoes.

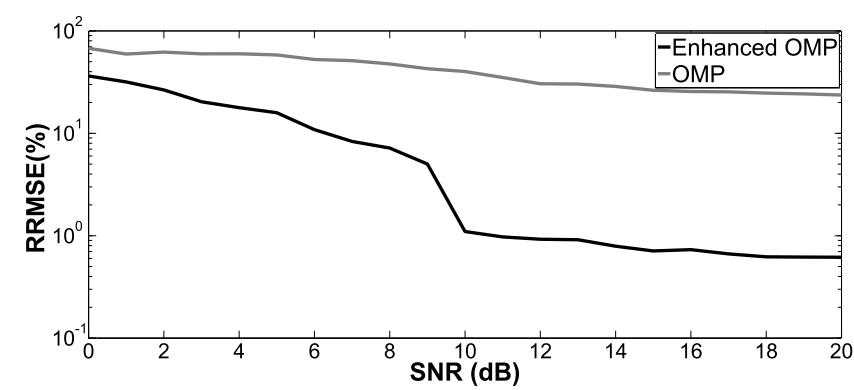

Fig. 10. Case 3, RRMSE on the estimated time of arrival $\hat{t}_{2}$ of weak signal $S_{2}$ versus SNR with 200 Monte-Carlo processes, overlapped echoes.

are considered. SNR varies from 0 to $20 \mathrm{~dB}$. According to simulation results, MUSIC algorithm fails to detect the echo backscattered from interface of similar layers. Consequently, only the results of conventional OMP are recorded as comparison in the following.

Figs. 9-10 plot the RRMSEs on the estimated time of arrival $\left(\hat{t}_{2}\right)$ of weak signal $S_{2}$ by the enhanced OMP and conventional OMP for Cases 2 and 3, respectively. As expected, for the proposed method, the RRMSEs continuously decrease with the increasing of SNR for both the overlapped and non-overlapped echoes. The proposed method has much smaller RRMSE than that of the conventional OMP at each SNR. Moreover, as shown in Fig. 10, the conventional OMP is with much higher RRMSEs in the scenario of overlapped echoes. To conclude, the proposed method outperforms the conventional OMP.

\section{EXPERIMENT}

In this section, the performance of the proposed enhanced OMP method is evaluated by the experimental data. An UWB step-frequency GPR made up of a vector network analyzer (VNA) and a bistatic antenna device (ETSA A5 antennas) is applied in the experiment. The distance between transmitter (Tx) and receiver ( $\mathrm{Rx})$ is approximately $20 \mathrm{~cm}$ (Fig. 12). The GPR is about $16.5 \mathrm{~cm}$ above the tested pavement. The radar frequency bandwidth ranges from $1.4 \mathrm{GHz}$ to $15.0 \mathrm{GHz}$, with $0.017 \mathrm{GHz}$ frequency step (801 frequency samples).

In the experiment, a two-layer asphalt pavement is studied as in Fig. 11. Its vertical structure and the experimental observation system is shown in Fig. 12. The studied pavement structure (pavement thickness is about $11 \mathrm{~cm}$ ) is made of an asphalt layer $\Sigma_{A}$ overlying another asphalt layer $\Sigma_{B}$, which is set on a metallic plane $\Sigma_{C}$. The thickness of the first layer is approximately $4 \mathrm{~cm}$ and the second layer is about $7 \mathrm{~cm}$.

\section{ACCEPTED MANUSCRIPT}




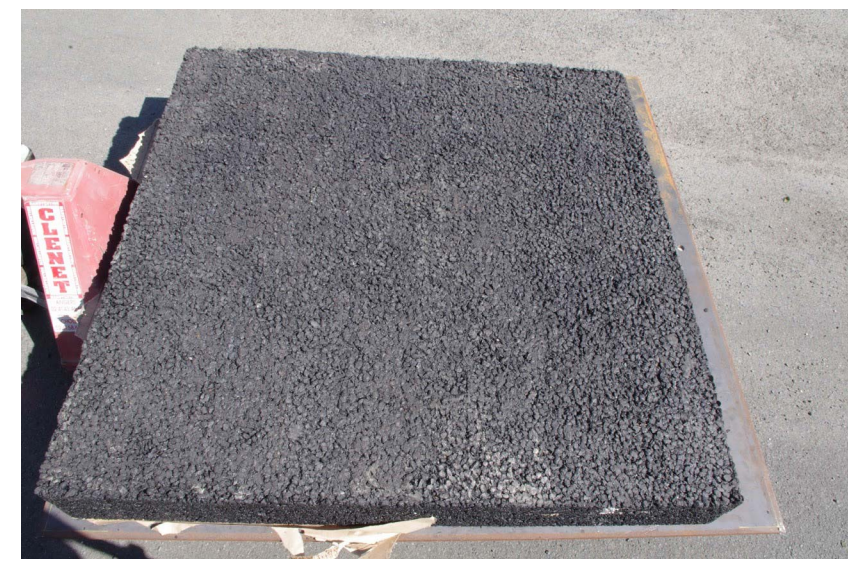

Fig. 11. Studied pavement.

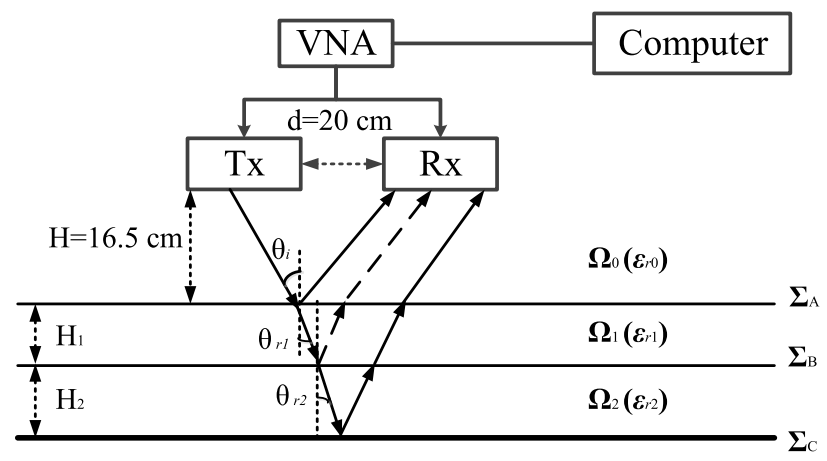

Fig. 12. Experimental device.

The length of the tested surface is about $80 \mathrm{~cm}$ with a sample step $4 \mathrm{~mm}$ (200 sample points). At each sample point, 10 snapshots are collected by using the observation system. The permittivities of the medium $\Omega_{2}$ and $\Omega_{3}$ are calculated from data [34], with $\varepsilon_{r 1}=4.93$ and $\varepsilon_{r 2}=5.16$. The incident wave with incidence angle $\theta_{i}=31.2^{\circ}$, then the refraction angles can be calculated with $\theta_{r 1}=13.5^{\circ}$ and $\theta_{r 2}=13.2^{\circ}$, respectively $\left(\sqrt{\varepsilon_{1}} \sin \theta_{1}=\sqrt{\varepsilon_{2}} \sin \theta_{2}\right)$.

\section{A. Data Set}

In real measurements, GPR moves slowly at different sample points of the pavement to generate independent spatial observations. Figs. 13a and 13b display the raw experimental data for both A-scan and B-scan, respectively. The black dashed lines represent the envelope of the received signals after the Hilbert transformation. It can be seen from Figs. 13a and $13 \mathrm{~b}$ that the raw data contains the multiple waves and air wave, which can be removed by using a time filter. The estimated echoes in Figs. 13a and 13b correspond to the first three backscattered echoes from three interfaces. The permittivities of medium $\Omega_{2}$ and $\Omega_{3}$ are very similar. Therefore, the echo from the second interface is very weak, whose amplitude is much smaller than that of the first and the third echoes. It is obvious that the first and third backscattered echoes are clearly visible in the figures, while the second echo is not clear and should be detected with some signal processing methods.

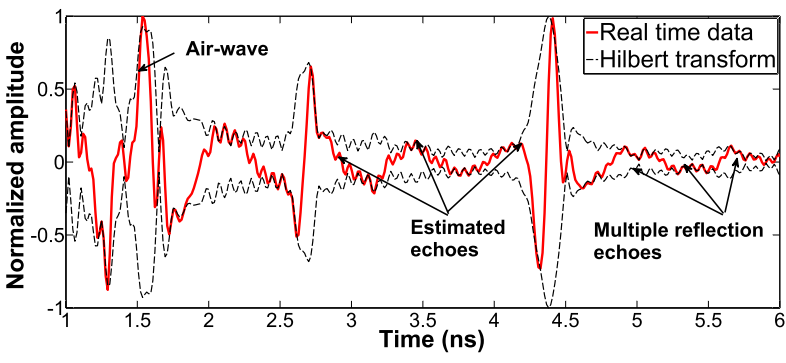

(a) Raw GPR data (A-scan)

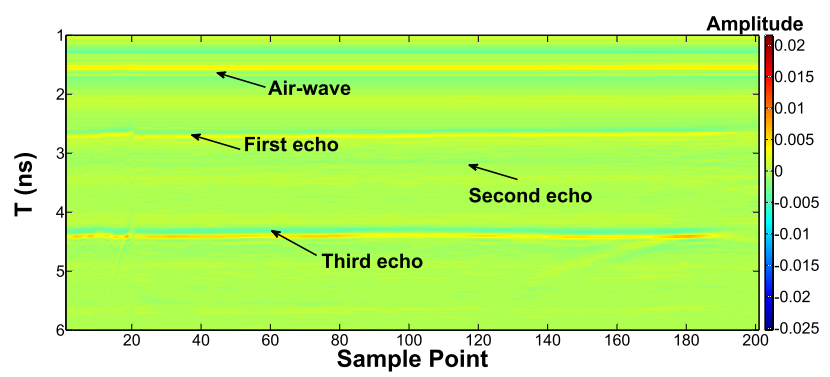

(b) Raw GPR data (B-scan)

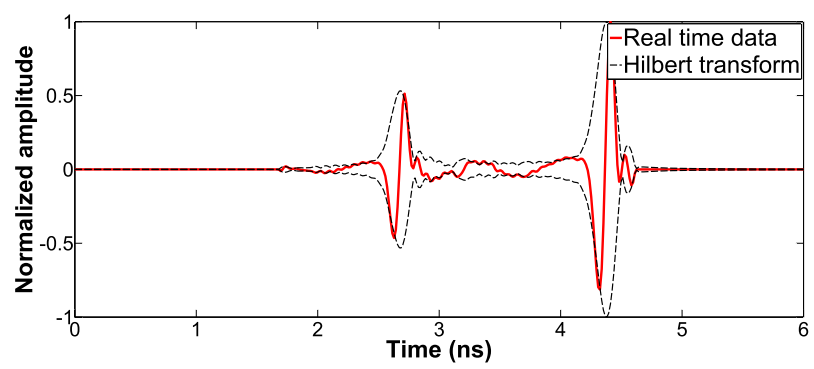

(c) Time filtered data

Fig. 13. Preprocessing of the backscattered echoes from the studied pavement.

\section{B. Pre-Processing of the Data}

The proposed enhanced OMP method cannot be applied without following preprocessing techniques: time filtering and data whitening.

1) Time Filtering. The aim of the time filtering preprocessing is to cut off the signals outside the GPR working time window (interested region) or to remove the residual of detected echoes. For example, as shown in Fig. $13 \mathrm{c}$, by applying time filtering, the multiple echoes and air wave can then be eliminated.

2) Data Whitening. The goal of data whitening is to remove the influence of radar pulse, which can be measured as the echo backscattered from a metallic plane.

Combined with the 2 preprocessing techniques, the proposed method can then be performed to detect the weak signal.

\section{Time-Delay and Thickness Estimation}

In the experiment, the enhanced OMP method is tested within the frequency bandwidth $B=[1.4,3.1] \mathrm{GHz}(101$ samples) in the scenario of overlapped echoes. The conventional OMP and MUSIC is taken as comparison. As illustrated in Fig. 14, three peaks corresponding to the first three backscattered echoes from three interfaces are clearly 


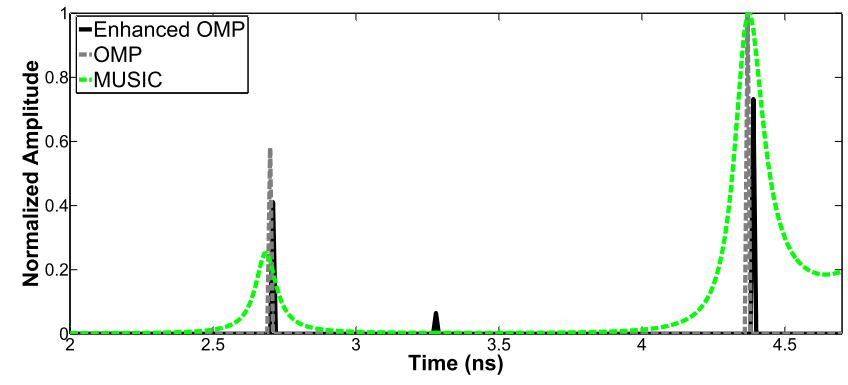

Fig. 14. Experiment results: estimated times of arrival of the backscattered echoes.

\section{TABLE III}

ESTIMATED TIME-DELAYS AND THICKNESSES By THE ENHANCED OMP METHOD, $\widehat{\Delta \tau}_{k}$ REPRESENTING THE Estimated TIME DELAY AND $\hat{H}_{k}$ REPRESENTING THE ESTIMATED THICKNESS

\begin{tabular}{|c|c|}
\hline Parameter $\quad$ Method & Modified OMP \\
\hline$\widehat{\Delta \tau_{1}}$ & $0.57 \mathrm{~ns}$ \\
\hline$\widehat{\Delta \tau}_{2}$ & $1.12 \mathrm{~ns}$ \\
\hline$\hat{H}_{1}$ & $3.75 \mathrm{~cm}$ \\
\hline$\hat{H}_{2}$ & $7.20 \mathrm{~cm}$ \\
\hline
\end{tabular}

observed by the proposed method, especially in the estimation of the second echo (weak signal). Afterwards, the times of arrival of three echoes can then be estimated. However, the conventional OMP and MUSIC fail to detect the weak signal.

Table III presents the estimated time-delays of the three echoes $\left(\widehat{\Delta \tau}_{1}\right.$ is the time-delay between the first and second echoes and $\widehat{\Delta \tau}_{2}$ is the time-delay between the second and third echoes). Consequently, the layer thickness can be calculated by following equation:

$$
\hat{H}_{k}=\frac{\widehat{c \Delta \tau_{k}} \cos \theta_{r k}}{2 \sqrt{\varepsilon_{r k}}}
$$

where $\hat{H}_{k}$ is the estimated thickness of the $k$ th layer with $k=1,2, c$ is the speed of light in vacuum, $\theta_{r k}$ is the refraction angle and $\varepsilon_{r k}$ is the permittivity of the $k$ th layer. The estimated layer thicknesses are also shown in the Table III. The proposed method provides an enhanced performance on thickness estimation as the estimated thicknesses are close to the real values.

\section{CONCLUSION}

This paper presents an enhanced OMP for automatic TDE and thickness estimation in the presence of thin thickness asphalt pavement, whose top layers have similar permittivity. The conventional methods cannot successful detect the weak signal backscattered from the interface of the top asphalt layers due to the small amplitude compared with the noise and other backscattered echoes. In contrast, the proposed method is able to handle the case of weak signals detection in the scenario of thin asphalt layers by suppressing the effect of noise and strong backscattered echoes. Consequently, the time-delays of backscattered echoes as well as layer thicknesses can be calculated. The proposed method is tested on both numerical and experimental data with an enhanced performance in TDE and thickness estimation compared with the conventional methods.

\section{REFERENCES}

[1] H. Guan, J. Li, Y. Yu, M. Chapman, and C. Wang, "Automated road information extraction from mobile laser scanning data," IEEE Trans. Intell. Transp. Syst., vol. 16, no. 1, pp. 194-205, Feb. 2015.

[2] F. Yang, L. Zhang, S. Yu, D. Prokhorov, X. Mei, and H. Ling, "Feature pyramid and hierarchical boosting network for pavement crack detection," IEEE Trans. Intell. Transp. Syst., vol. 21, no. 4, pp. 1525-1535, Apr. 2020.

[3] Y. Zhang et al., "A kinect-based approach for 3D pavement surface reconstruction and cracking recognition," IEEE Trans. Intell. Transp. Syst., vol. 19, no. 12, pp. 3935-3946, Dec. 2018.

[4] M. Sun, J. Pan, C. Le Bastard, Y. Wang, and J. Li, "Advanced signal processing methods for ground-penetrating radar: Applications to civil engineering," IEEE Signal Process. Mag., vol. 36, no. 4, pp. 74-84, Jul. 2019.

[5] M. Ambrosanio, M. T. Bevacqua, T. Isernia, and V. Pascazio, "The tomographic approach to ground-penetrating radar for underground exploration and monitoring: A more user-friendly and unconventional method for subsurface investigation," IEEE Signal Process. Mag., vol. 36, no. 4, pp. 62-73, Jul. 2019.

[6] X. Liu et al., "Ground penetrating radar (GPR) detects fine roots of agricultural crops in the field," Plant Soil, vol. 423, nos. 1-2, pp. 517-531, Feb. 2018.

[7] A. Benedetto and L. Pajewski, Civil Engineering Applications of Ground Penetrating Radar (Springer Transactions in Civil and Environmental Engineering). Cham, Switzerland: Springer, 2015.

[8] D.-H. Chen, F. Hong, W. Zhou, and P. Ying, "Estimating the hotmix asphalt air voids from ground penetrating radar," NDT \& E Int., vol. 68, pp. 120-127, Dec. 2014.

[9] D. Kurrant and E. Fear, "Technique to decompose near-field reflection data generated from an object consisting of thin dielectric layers," IEEE Trans. Antennas Propag., vol. 60, no. 8, pp. 3684-3692, Aug. 2012.

[10] J. S. Lee, C. Nguyen, and T. Scullion, "A novel, compact, lowcost, impulse ground-penetrating radar for nondestructive evaluation of pavements," IEEE Trans. Instrum. Meas., vol. 53, no. 6, pp. 1502-1509, Dec. 2004.

[11] W. W.-L. Lai, X. Dérobert, and P. Annan, "A review of ground penetrating radar application in civil engineering: A 30-year journey from locating and testing to imaging and diagnosis," NDT \& E Int. vol. 96, pp. 58-78, Jun. 2018.

[12] J. Pan, C. Le Bastard, Y. Wang, and M. Sun, "Time-delay estimation using ground-penetrating radar with a support vector regression-based linear prediction method," IEEE Trans. Geosci. Remote Sens., vol. 56, no. 5, pp. 2833-2840, May 2018.

[13] S. Wang, S. Zhao, and I. L. Al-Qadi, "Real-time density and thickness estimation of thin asphalt pavement overlay during compaction using ground penetrating radar data," Surv. Geophys., vol. 41, pp. 1-15, Jul. 2019.

[14] S. Wang, S. Zhao, and I. L. Al-Qadi, "Continuous real-time monitoring of flexible pavement layer density and thickness using ground penetrating radar," NDT \& E Int., vol. 100, pp. 48-54, Dec. 2018.

[15] H. Liu and M. Sato, "In situ measurement of pavement thickness and dielectric permittivity by GPR using an antenna array," NDT \& E Int., vol. 64, pp. 65-71, Jun. 2014.

[16] C. Le Bastard, V. Baltazart, Y. Wang, and J. Saillard, “Thin-pavement thickness estimation using GPR with high-resolution and superresolution methods," IEEE Trans. Geosci. Remote Sens., vol. 45, no. 8, pp. 2511-2519, Aug. 2007.

[17] J. Pan, M. Sun, Y. Wang, C. L. Bastard, and V. Baltazart, "A time-delay estimation approach for coherent GPR signals by taking into account the noise pattern and radar pulse," Signal Process., vol. 176, Nov. 2020, Art. no. 107654.

[18] S. Zhao and I. L. Al-Qadi, "Super-resolution of 3-D GPR signals to estimate thin asphalt overlay thickness using the XCMP method," IEEE Trans. Geosci. Remote Sens., vol. 57, no. 2, pp. 893-901, Feb. 2019.

[19] M. Sun, C. Le Bastard, Y. Wang, and N. Pinel, "Time-delay estimation using ESPRIT with extended improved spatial smoothing techniques for radar signals," IEEE Geosci. Remote Sens. Lett., vol. 13, no. 1, pp. 73-77, Jan. 2016.

[20] L. Qu, Q. Sun, T. Yang, L. Zhang, and Y. Sun, "Time-delay estimation for ground penetrating radar using ESPRIT with improved spatial smoothing technique," IEEE Geosci. Remote Sens. Lett., vol. 11, no. 8, pp. 1315-1319, Aug. 2014.

\section{ACCEPTED MANUSCRIPT}


[21] M. Sun, Y. Wang, C. Le Bastard, J. Pan, and Y. Ding, "Signal subspace smoothing technique for time delay estimation using MUSIC algorithm," Sensors, vol. 17, no. 12, p. 2868, Dec. 2017.

[22] J. Li, C. Le Bastard, Y. Wang, G. Wei, B. Ma, and M. Sun, "Enhanced GPR signal for layered media time-delay estimation in low-SNR scenario," IEEE Geosci. Remote Sens. Lett., vol. 13, no. 3, pp. 299-303, Mar. 2016.

[23] J. Pan, M. Sun, Y. Wang, C. Le Bastard, and V. Baltazart, "Timedelay estimation by a modified orthogonal matching pursuit method for rough pavement," IEEE Trans. Geosci. Remote Sens., vol. 59, no. 4, pp. 2973-2981, Apr. 2021.

[24] N. Kim, S. Kim, Y.-K. An, and J.-J. Lee, "Triplanar imaging of 3-D GPR data for deep-learning-based underground object detection," IEEE J. Sel. Topics Appl. Earth Observ. Remote Sens., vol. 12, no. 11, pp. 4446-4456, Nov. 2019.

[25] Z. Tong, D. Yuan, J. Gao, Y. Wei, and H. Dou, "Pavement-distress detection using ground-penetrating radar and network in networks," Construct. Building Mater, vol. 233, Feb. 2020, Art. no. 117352.

[26] Y. Ji et al., "Deep neural network-based permittivity inversions for ground penetrating radar data," IEEE Sensors J., vol. 21, no. 6, pp. 8172-8183, Mar. 2021.

[27] Z. Tong, J. Gao, and D. Yuan, "Advances of deep learning applications in ground-penetrating radar: A survey," Construct. Building Mater. vol. 258, Oct. 2020, Art. no. 120371.

[28] Z. Leng and I. L. Al-Qadi, "An innovative method for measuring pavement dielectric constant using the extended CMP method with two air-coupled GPR systems," NDT \& E Int., vol. 66, pp. 90-98, Sep. 2014.

[29] S. Zhao and I. L. Al-Qadi, "Development of regularization methods on simulated ground-penetrating radar signals to predict thin asphalt overlay thickness," Signal Process., vol. 132, pp. 261-271, Mar. 2017.

[30] Z. Dong et al., "Rapid detection methods for asphalt pavement thicknesses and defects by a vehicle-mounted ground penetrating radar (GPR) system," Sensors, vol. 16, no. 12, p. 2067, Dec. 2016.

[31] D. Batrakov, M. Antyufeyeva, K. Beloshenko, A. Batrakova, and V. Troyanovsky, "GPR signals processing for the road pavements monitoring with the weak contrast layers," in Proc. Eur. Microw. Conf. Central Eur., 2019, pp. 390-393.

[32] C. Fauchard, "Utilisation de radars très hautes fréquences: Application à l'auscultation non destructive des chaussées," Ph.D. dissertation, Dept. Lab. Central Ponts Chaussées, Univ. Nantes, Nantes, France, 2001.

[33] D. J. Daniels, Ground Penetrating Radar, 1st ed. Edison, NJ, USA: IET, 2004.

[34] I. L. Al-Qadi and S. Lahouar, "Measuring layer thicknesses with GPR-Theory to practice," Construct. Building Mater, vol. 19, no. 10, pp. 763-772, Dec. 2005.

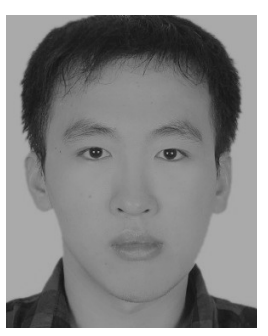

Meng Sun received the B.S. degree from Northwest University in 2010, the M.S. degree from the South China University of Technology, China, in 2013, and the Ph.D. degree from the IETR Laboratory, Polytech Nantes, Université de Nantes, France, in 2016. $\mathrm{He}$ is currently with the Department of Electronic Engineering, Nanjing University of Aeronautics and Astronautics, Nanjing, China. His main research interest includes signal processing techniques on parameter estimation of civil engineering materials.

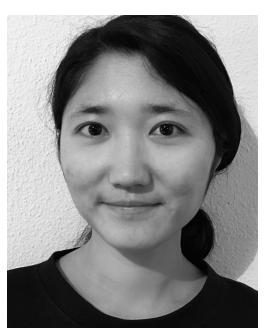

Jingjing Pan (Member, IEEE) received the B.S. degree in cartography and geographic information science from East China Normal University in 2012, the M.S. degree in cartography and geographic information science from Beijing Normal University in 2015, and the Ph.D. degree from the Polytech Nantes, Université de Nantes, France, in 2019. She is currently a Post-Doctoral Fellow with the Department of Electronic Engineering, Nanjing University of Aeronautics and Astronautics, Nanjing, China. Her research interests include array signal processing, ground penetrating radar, and nondestructive testing.

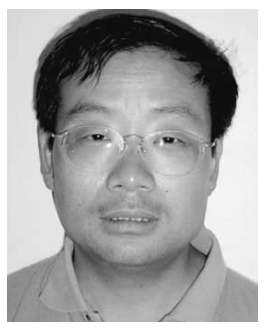

Yide Wang (Senior Member, IEEE) received the B.S. degree in electrical engineering from the Beijing University of Posts and Telecommunications, Beijing, China, in 1985, and the M.S. and $\mathrm{Ph} . \mathrm{D}$. degrees in signal processing and telecommunications from the University of Rennes 1, France, in 1986 and 1989, respectively.

$\mathrm{He}$ is currently a Professor with the Ecole Polytechnique de l'Université de Nantes. His research interests include array signal processing, spectral analysis, and mobile wireless communication systems.

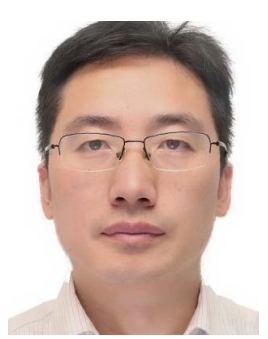

Xiaofei Zhang received the M.S. degree from Wuhan University, Wuhan, China, in 2001, and the $\mathrm{Ph} . \mathrm{D}$. degree in communication and information systems from the Nanjing University of Aeronautics and Astronautics, Nanjing, China, in 2005. He is currently a Professor with the Department of Electronic Engineering, Nanjing University of Aeronautics and Astronautics. His research interests include array signal processing and communication signal processing.

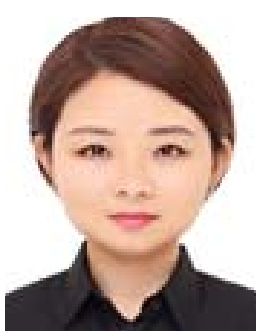

Xiaoting Xiao received the B.Eng. degree in electronic information engineering from the Chongqing University of Posts and Telecommunications, China, in 2010, the M.Eng. degree from the Polytech Nantes, France, in 2012, and the Ph.D. degree in electronic, microelectronic, nanoelectronic, and microwaves from Nantes University and the French Institute of Science and Technology for Transport, Development and Networks, France, in 2016. She worked as a Post-Doctoral Fellow with the University of Electronic Science and Technology of China from 2016 to 2020. She subsequently joined Southwest Petroleum University. She is working in the field of electromagnetic non-destructive testing, especially ground penetrating radar. Her research interests include the electromagnetic modeling, imaging algorithms, and the inversion methods for high resolution non-destructive characterization of civil engineering materials.

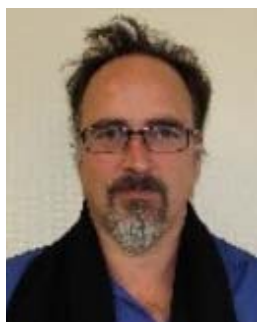

Cyrille Fauchard received the B.S. and M.S degrees in material science and the Ph.D. degree in electronic engineering from L'Université Nantes Angers, Le Mans (LUNAM), France, in 1993, 1995, and 2001, respectively. His research interests include near surface geophysics and electromagnetic characterization of civil engineering and earth materials.

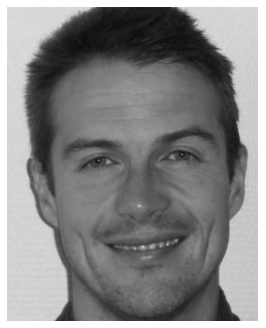

Cédric Le Bastard received the B.S. and M.S. degrees in electronic engineering from the University of Rennes 1, Rennes, France, in 2001 and 2003 , respectively, and the Ph.D. degree from the Central des Ponts et Chaussées Laboratory, University of Nantes, Nantes, France, in 2007. His research interests include radar, nondestructive testing and evaluation, electromagnetic wave scattering, time-delay estimation, and array signal processing. 